\title{
Prospective study of ventilated patients in the paediatric medical intensive care unit of Lady Ridgeway Hospital
}

\author{
Thadchanamoorthy Vijayakumary ${ }^{1}$, J R Srilal de Silva ${ }^{2}$, Jeevani Sarathchandra ${ }^{3}$, Balachandran \\ Kumarendran ${ }^{4}$
}

Sri Lanka Journal of Child Health, 2012; 41(3): 114-117

\begin{abstract}
Objective: To determine the characteristics and outcome of ventilated patients in the paediatric medical intensive care unit (PICU) of Lady Ridgeway Hospital for Children (LRH), Colombo.
\end{abstract}

Design: Prospective descriptive study

Method: The study population consisted of all ventilated paediatric patients admitted to PICU, LRH from $1^{\text {st }}$ March to $31^{\text {st }}$ August, 2009. The factors studied included demographic profile (age, sex, residence, transfer), length of stay on ventilator, indication for ventilation, details of organ dysfunction and the final outcome.

Results: There were 152 patients ventilated over the study period of six months. Seventy three were under one year of age. Male to female ratio was $1.17: 1$. A significant proportion $(n=105)$ were from the Western province. Eighty five patients were transferred from medical wards of LRH. Median duration of ventilation was six days (IRQ 4-10). Indications for ventilation included respiratory problems (85), neurological disorders (13), cardiac causes (5), hepatic problem (1) and miscellaneous diseases (48) including 32 from dengue syndromes. There were 42 deaths among ventilated patients giving an overall mortality rate of $27.6 \%$. Multiorgan dysfunction syndrome (MODS) on admission accounted for $81 \%$ of the deaths. Bronchopneumonia (16) and dengue haemorrhagic fever (12) were the main underlying causes of death. Thirty two $(21 \%)$ had anaemia while in the PICU, 15 of them having anaemia on admission. Nosocomial infection was present in 17 (11\%) patients. Children transferred from other provinces had a higher risk of death than those from the Western province $(\mathrm{OR}=1.5,95 \% \mathrm{CI}: 0.7-3.3)$.

${ }^{1}$ Senior Registrar, ${ }^{2}$ Consultant Paediatrician, PICU, ${ }^{3}$ Consultant Paediatrician, Lady Ridgeway Hospital for Children, Colombo, ${ }^{4}$ Lecturer, Department of Public Health, Faculty of Medicine, University of Kelaniya, Ragama

(Received on 11 September 2011; Accepted after revision on 28 October 2011)
Conclusions: MODS on admission accounted for $81 \%$ of the deaths. Bronchopneumonia and dengue haemorrhagic fever were the main underlying causes of death. Children transferred from other provinces had a higher risk of death compared to those from the Western province

(Key words: Medical intensive care; nosocomial infection; anaemia; mortality)

\section{Introduction}

A decline in mortality from various diseases is ensured by well-developed hospital care including services in intensive care units. Numerous conditions that were previously fatal are now treatable $^{1}$. Children today are born healthier but more and more are also being admitted to hospital than ever before ${ }^{2}$. In the context of intensive care management, outcome analysis coupled with demographic status and clinical details have become a challenge for the modern day intensivist ${ }^{3}$. There are many studies documenting outcomes of paediatric intensive care units from Western countries $^{4,5,6}$ but very few studies are available from developing countries ${ }^{7}$. The Lady Ridgeway Hospital for Children (LRH) is the 1100 bedded paediatric teaching hospital to the Faculty of Medicine, Colombo and one of the two specialised tertiary care hospitals in Sri Lanka. Ten beds have been allocated to the paediatric medical intensive care unit (PICU). The PICU is staffed by two consultants (a paediatrician and an intensivist), five to six paediatric senior registrars and eight senior medical officers. They are responsible for covering the PICU 24 hours a day and handling ventilators with back up support from biomedical engineers. Nurse to patient ratio is $1: 1$. Admission age in our PICU is 12 years and below based on existing hospital regulations. Admission of a child to PICU is decided by the consultants. The number of patients ventilated in the PICU averages 20 to 25 per month. Although the PICU, LRH delivers yeomen services to children, there is no information available regarding the variety of clinical conditions, epidemiological profile and outcome of patients in this unit. The current study is intended to provide an assessment of the baseline status at the present time and could be used for 
comparison with future studies and also serve as a yardstick to compare the current status against the results of other paediatric intensive care units.

\section{Objective}

To determine the characteristics and outcome of ventilated patients in the PICU, LRH.

\section{Method}

A prospective descriptive clinical study was conducted in the PICU, LRH. We consecutively collected data from Bed Head Tickets (BHTs) of all ventilated paediatric patients till discharge or death from $1^{\text {st }}$ March to $31^{\text {st }}$ August 2009, a period of six months. Those patients who stayed less than two hours in the PICU and neonates were excluded. Re-admissions to the PICU during the same hospitalization were analyzed as separate patients, as there was a possibility of a different outcome at each admission. All patients admitted for invasive ventilation during the study period were included irrespective of the source of admission i.e. transfer from a peripheral hospital or from LRH itself. Data collected on patients included the demographic profile (age, gender, province patient belongs to, admission source) length of stay on ventilator, reasons for ventilation, outcome of patients (either death or discharge) and organ involvement, with the involvement of 3 or more organs being defined as multi-organ dysfunction syndrome (MODS) $)^{8,9}$. Data was collected into a purpose designed questionnaire by the principal investigator and entered into EpiInfo software and analyzed using SPSS and R statistical.

\section{Results}

Of the 292 patients admitted to PICU during the study period, $152(52 \%)$ were ventilated. Of the ventilated patients, 73 were under one year, 56 between $1-5$ years and 23 more than 5 years. The male to female ratio was $1.17: 1$. Of the patients, $105(69 \%)$ had their residence in the Western province, followed by $14(9 \%)$ in the Southern province, and $12(8 \%)$ in the Subaragamuwa province. Eighty five patients were directly admitted from the paediatric medical wards of LRH while the rest were transferred from peripheral hospitals. Median duration of ventilation was 6 days (IRQ 4-10). Males needed a slightly more prolonged period of intubation than females (Mann-Whitney $U$ test, $p=0.43$ ). The indications for ventilation are shown in table 1 . Among the miscellaneous causes dengue syndromes accounted for 32 admissions.
Table-1

Indications for ventilation

\begin{tabular}{|l|c|c|}
\hline \multicolumn{1}{|c|}{ Indication } & Number & \% \\
\hline Respiratory & 85 & $(56 \%)$ \\
\hline Neurological & 13 & $(8.5 \%)$ \\
\hline Cardiovascular & 5 & $(3.3 \%)$ \\
\hline Hepatic & 1 & $(0.7 \%)$ \\
\hline Miscellaneous & 48 & $(31.5 \%)$ \\
\hline
\end{tabular}

On admission to the PICU, $96(63 \%)$ patients had multi-organ involvement; 49 (32\%) patients needed vascular support with one or more fluid boluses while 52 (34\%) needed dopamine as an inotrope; $15(10 \%)$ patients required blood transfusions; 88 $(58 \%)$ patients had infection with associated disseminated intravascular coagulation (DIC) in 32 $(21 \%)$ of them. After admission, $32(21 \%)$ patients were transfused blood and $105(69 \%)$ had infection with DIC in $41(27 \%)$ of them. Fourteen $(9 \%)$ ventilated patients had one cardiac arrest before admission, 12 (8\%) needing cardiopulmonary resuscitation with drugs and 3 (2\%) needing desynchronized cardioversion. Nosocomial infection was present in $17(11 \%)$ patients. There were 42 deaths giving an overall mortality rate of $27.6 \%$. The ultimate cause of death in most of the patients, irrespective of the underlying problem, was MODS on admission accounting for $34(81 \%)$ deaths. The underlying causes among the nonsurvivors are shown in table 2.

Table-2

Underlying causes among non-survivors

\begin{tabular}{|c|c|}
\hline Cause & Frequency (\%) \\
\hline Bronchopneumonia & $16(38)$ \\
\hline $\begin{array}{l}\text { Dengue haemorrhagic } \\
\text { fever grade IV }\end{array}$ & $12(28.5)$ \\
\hline Encephalitis & $4(9.5)$ \\
\hline Myocarditis & $1(2.4)$ \\
\hline Dengue encephalitis & $1(2.4)$ \\
\hline Acute leukaemia & $1(2.4)$ \\
\hline $\begin{array}{l}\text { Complex congenital } \\
\text { heart disease }\end{array}$ & $1(2.4)$ \\
\hline Tuberculous meningitis & $1(2.4)$ \\
\hline Renal tubular acidosis & $1(2.4)$ \\
\hline Neuroblastoma & $1(2.4)$ \\
\hline Kikuchi disease & $1(2.4)$ \\
\hline Intracranial haemorrhage & $1(2.4)$ \\
\hline Lobar pneumonia & $1(2.4)$ \\
\hline Total & $42(100)$ \\
\hline
\end{tabular}


Children from other provinces had a higher risk of death than those from the Western province $(\mathrm{OR}=1.5$, 95\% CI: 0.7-3.3). Patients admitted directly from medical wards of LRH (internal transfers) accounted for $27 \%$ of mortality in comparison to those transferred from peripheral hospitals (external transfers) (28\%). This was not statistically significant.

\section{Discussion}

Modern paediatric intensive care is characterized by increased sophistication, resulting in spiralling costs. Auditing the PICU is thus an integral component in health planning and management. There is a need to accurately define prognosis, so that physicians can be guided in clinical decision making, including the appropriateness of therapy ${ }^{10,11}$. Moreover, the impact of new technologies and medical intervention can be assessed in a more objective fashion. A gradual rise in the incidence of epidemiological diseases like dengue, improving socioeconomic status and increasing awareness of health related issues among urban population have led to increasing demand for paediatric critical care in Sri Lanka. Studies in developing countries like India and Pakistan have reported significant variability between PICUs in age and percentage of morbidity and mortality ${ }^{1,6}$. PICUs with higher mortality rates may be caring for patients with more severe illnesses and vice versa. However, lower mortality rates do not necessarily translate into better long term outcomes ${ }^{6}$.

In the present study we have encountered a wide spectrum of cases requiring inotropes and various critical care treatment modalities such as renal and hepatic support. Due to lack of publication of PICU profile within Sri Lanka, an adequate comparison of relative incidence and outcomes is not possible within the country. Comparison within developing countries is however feasible A prospective study in a PICU in New Delhi, India ${ }^{6}$ and a retrospective study in a PICU at the Aga Khan University Hospital in Pakistan ${ }^{1}$ have shown that males are preponderant in their PICU population and that respiratory disease was the most common cause for ventilation. We found similar results in both parameters. In our study, the second most common cause for ventilation was dengue $(21 \%)$, but in India dengue accounted for only $5.5 \%$ of cases ${ }^{6}$. The duration of mechanical ventilation was $6.39+/-$ 1.2 days in India and 6 days (IRQ 4-10) in our study.

During PICU stay nosocomial infection is a critical problem $^{12,13}$. This could be due to blood stream infection from central venous catheters (mostly placed in the femoral vein) and ventilator associated pneumonias. Nosocomial infection accounted for $11 \%(17 / 152)$ in our study, which is higher than recent studies in developing countries ${ }^{1}$. This variability is most likely due to noncompliance with infection control measures, but it is within the range of $5-15 \%$ in some developed countries $^{14}$ and better than other reported PICU studies from Pakistan ${ }^{4,15}$.

Anaemia is common in critically ill children admitted to the PICU. There are several possible causes for the anaemia of critical illness, including chronic anaemia, overt and occult blood loss, underlying disease treatment causing bone marrow suppression and iatrogenic bleeding for investigations. Our study showed that $10 \%$ of ventilated patients were anaemic on admission and $21 \%$ had anaemia while in the PICU compared to the study done in the PICU, North America ${ }^{16}$ where $33 \%$ of patients had anaemia on admission and $41 \%$ developed it while in the ICU.

Overall mortality was $27.6 \%$ with the age related mortality being highest (43.5\%) in age group more than 5 years which is dissimilar in comparison to other studies ${ }^{6}$. Patients transferred from hospitals which are far away from LRH had a higher mortality compared to those who were admitted from medical wards, LRH. This is probably due to poor status on admission. It is difficult to compare our mortality rate with other developing and developed countries since they have compared mortality against total PICU admissions including both ventilated and non-ventilated patients. An unpublished report in PICU, Pakistan had mortality rates which ranged from $25-40 \%$ from 1993 to $2005^{1}$ and published data in 2007 had $22-29 \%{ }^{4,15}$. However, a recent one year retrospective study in 2007 showed a $14 \%$ mortality ${ }^{1}$.

Among non-survivors, MODS on admission accounted for $81 \%$ of deaths in our study while $49.5 \%$ of non-survivors had MODS in India ${ }^{6}$. MODS is as much a therapeutic challenge in Sri Lanka as in India $^{17}$.

\section{Conclusions}

- MODS on admission accounted for $81 \%$ of deaths in our study.

- Bronchopneumonia and dengue haemorrhagic fever were the main underlying causes of death.

- Children from other provinces had a higher risk of death compared to those from the Western province $(\mathrm{OR}=1.5,95 \% \mathrm{CI}: 0.7-$ 3.3). 
While this audit identifies the risk factors for death, it also provides a baseline to monitor the improvement of the clinical outcome of the PICU in LRH.

\section{Acknowledgements}

We thank Dr. B J C Perera for his invaluable guidance and advice in every aspect of this study. We also acknowledge PICU staff for their cooperation during the study period.

\section{References}

1. Haque A, Bano S. Clinical profile and outcome in a paediatric intensive unit in Pakistan. Journal of the College of Physicians and Surgeons of Pakistan 2009; 19(8):534-5.

2. Esmal A, Quaylle J. Assessing the appropriateness of paediatric hospital admission in the United Kingdom. Journal of Public Health Medicine 2000; 22(2): 231-8. http://dx.doi.org/10.1093/pubmed/22.2.231

3. Tan GH, Tan TH, Goh DYT. Risk factors for predicting in a paediatric intensive care unit. Annals Academy of Medicine Singapore 1998; 27:813-8.

4. Khan HI, Khaliq N, Afzal MF. Paediatric intensive care unit: Pattern of admissions. Professional Medical Journal 2006; 13:35861.

5. Qureshi AU, Ali AS, Ahamad TM. Comparison of three prognostic scores (PRISM, PELOD and PIMZ) at paediatric intensive care unit under Pakistanis circumstances. Journal of Ayub Medical College Abbottabad 2007; 19:49-53.

6. Khilnani P, Sarma D, Singh R. Demographic profile and outcome analysis of a tertiary paediatric intensive care unit. Indian Journal of Pediatrics 2004; 71(7):587-91. http://dx.doi.org/10.1007/BF02724117

7. Fiser D H. Assessing the outcome of paediatric intensive care. Journal of Pediatrics 1992; 121:68-74. http://dx.doi.org/10.1016/S00223476(05)8254 $\underline{4-2}$

8. Goldstein B, Giroir B. International paediatric sepsis consensus conference: Definition for sepsis and organ dysfunction in paediatrics. Pediatric Critical Care Medicine 2005; 6(1):2-8. http://dx.doi.org/10.1097/01.PCC.0000149131. $\underline{\text { 72248.E6 }}$
9. Wilkinson JD, Pollack MM, Glass NL. Mortality associated with multiple organ system failure and sepsis in paediatric intensive care unit. Journal of Pediatrics 1987; 111:324-28. http://dx.doi.org/10.1016/S00223476(87)8044 $\underline{8-1}$

10. Knaus WA, Wagner DP, Lynn J. Short term mortality predictions for critically ill hospitalized adult: science and ethics. Science 1991; 254:389-94. http://dx.doi.org/10.1126/science.1925596

11. Sachdeva RC, Jerfferson LS, Coss-Bu JR. Resource consumption and the. extent of futile care among patients in a paediatric intensive care unit setting. Journal of Pediatrics 1996; 128:742-7. http://dx.doi.org/10.1016/S00223476(96)7032 $\underline{3-2}$

12. Dereka A. Epidemiology of severe sepsis in the United States: Analysis of incidence, outcome and associated cost of care. Critical Care Medicine 2001; 29(7):1303-10. http://dx.doi.org/10.1097/00003246200107000 $\underline{-00002}$

13. Yogaraj JS, Edward AM, Victoria J. Rate, risk factors, and outcome of nosocomial primary bloodstream infection in paediatric intensive care unit patients. Pediatrics 2002; 110(3): 481-3. http://dx.doi.org/10.1542/peds.110.3.481

14. Correia M, Smano C, Lito LM. Nosocomial infection in a paediatric intensive care unit. Acta Medica Portuguesa 1977; 10(6-7):463-8.

15. Hamid MH, Zafar A, Moqbool S. Nosocomial blood stream infection in a tertiary care paediatric intensive care unit. Journal of the College of Physicians and Surgeons of Pakistan 2007; 17:416-9.

16. Bateman ST, Lacroix J, Boven K. Anaemia, blood loss, and blood transfusion in North American children in the intensive care unit. American Journal of Respiratory Critical Care Medicine 2008; 178:26-33. http://dx.doi.org/10.1164/rccm.2007111637OC

17. Khilnani P, Sarma D. Epidemiology \& peculiarities of paediatric multi-organ dysfunction syndrome in New Delhi, India. Intensive Care Medicine 2006; 32(11):1856-2. http://dx.doi.org/10.1007/s00134-006-0373-5 